Tristán Sarmiento, R., Serrano Pastor, F. J. y Martínez Segura, M. J. (2021). Influencia de la implicación familiar eñ lōs deberes escolares en Educación Primaria. Percepción de la comunidad educativa en centros de la Región de Murcia. Revista de Investigación Educativa, 39(2), 335-350.

DOI: http://dx.doi.org/10.6018/rie.363891

\title{
Influencia de la implicación familiar en los deberes escolares en Educación Primaria. Percepción de la comunidad educativa en centros de la Región de Murcia
}

\author{
Family involvement influence in Primary Education homework. \\ Perception of the educational community in centers of the Region \\ of Murcia
}

\author{
Ruth Tristán Sarmiento*, Francisca José Serrano Pastor** y María José Martínez Segura** \\ *Escuela Internacional de Doctorado. Facultad de Educación. Universidad de Murcia (España) \\ **Departamento de Métodos de Investigación y Diagnóstico en Educación. Facultad de Educación. Universidad de \\ Murcia (España)
}

\begin{abstract}
Resumen
Este trabajo tiene como objetivo identificar las percepciones de la comunidad educativa del segundo tramo de Educación Primaria de centros de la Región de Murcia sobre la influencia que tiene la implicación familiar en la realización de los deberes escolares. La recogida de información se ha llevado a cabo mediante tres cuestionarios estructurados, elaborados ad hoc y destinados a cada uno de los grupos participantes. Tales grupos han estado conformados por 259 estudiantes, 81 docentes y 163 familias.

El diseño de la investigación y la naturaleza de la información han requerido un tratamiento cuantitativo de carácter descriptivo, mediante un estudio de encuesta. Previamente al análisis de los resultados, también se ha estudiado la consistencia interna y la validez de constructo de las escalas de los cuestionarios en torno a la dimensión implicación familiar y deberes escolares. Entre los resultados se evidencia la existencia de una percepción positiva por parte de toda la comunidad hacia la participación de los progenitores en las tareas escolares, ya que piensan que dicha colaboración aporta grandes beneficios a los estudiantes.
\end{abstract}

Palabras clave: deberes; familia; personal docente; alumno.

Correspondencia: Ruth Tristán Sarmiento, ruth.tristan@um.es, Facultad de Educación, 9, Campus Universitario de Espinardo, Universidad de Murcia, 30100, Murcia. 


\begin{abstract}
This study examines the opinions on the influence of family involvement in homework completion of the educational community in the second cycle of Primary Education at schools in Murcia. The collection of data has been carried out through three structured, ad-hoc questionnaires intended for each of the participating groups. These groups consist of 259 students, 81 teachers and 163 families; 503 participants in total.

The design of this research and the nature of the collected information require a quantitative analysis, descriptive in nature, that has been conducted as a survey study. Prior to the analysis of the results, the internal consistency and construct validity of the questionnaire's scales regarding family involvement in/and homework completion have been studied. The findings demonstrate the existence of a positive perception shared by the whole community towards the participation of parents in school tasks, since such collaboration is believed to provide great benefits for schoolchildren.

Keywords: homework; family; teaching staff; student.
\end{abstract}

\title{
Introducción
}

Esta investigación tiene como objetivo principal averiguar cuáles son las percepciones de la comunidad educativa de nueve centros de Educación Primaria de la Región de Murcia sobre la implicación de las familias en la realización de los deberes escolares (en adelante, DE).

Los datos plasmados en el informe PISA de 2012 revelan que España se encuentra entre los países de la OCDE con más volumen de DE sin que ello redunde en unos mejores resultados académicos (Bailén y Polo, 2016). Además, la relevancia de esta problemática ha tenido y tiene un gran impacto en los órganos de gobierno de nuestro país. Prueba de ello es que en 2016 el Congreso de los Diputados instara al Gobierno a regular los deberes por ley para reconocer el derecho de los escolares a la disposición de manera efectiva de su tiempo libre. Recientemente, en la Comunidad Valenciana se ha aprobado la Ley 26/2018, de 21 de diciembre, de la Generalitat, de derechos y garantías de la infancia y adolescencia, en la que se limitan las tareas escolares para casa. En diciembre del mismo año, el Gobierno de Aragón publicó una orden donde se solicitaba a la reflexión sobre los DE. Por su lado, la Asamblea Regional de Murcia instó en 2016 al Consejo de Gobierno a la regulación y racionalización de los DE en horario extraescolar con la propuesta de diversas medidas entre las que se encuentra la solicitud a las universidades de la "introducción de contenidos y formación específica en materia de deberes escolares” (p. 2401).

Es destacable que, en países como EEUU, el tema de la importancia de los DE es un aspecto que se ha estado debatiendo durante más de 100 años, siendo prohibidos en ciertas épocas y elogiados en muchas otras (Blazer, 2009). También fueron cuestionados en Colombia durante toda la mitad del siglo XX, donde eran entendidos por algunas corrientes como actividades disciplinares; y como tareas que acercaban a los alumnos al conocimiento por otros (Jiménez Becerra, 2010).

En cuanto a España, este tema también produjo debates cíclicos en lo referido a su valoración; pero finalmente, parecía que se habían convertido en algo que todo el mundo tenía asimilado. Era una tradición arraigada, así que nadie objetaba nada al respecto. Pero, hace unos años, esto cambió y empezaron a ser cuestionados tanto por 
familiares, como por docentes; y entre los motivos se destacaban las nuevas estructuras sociales o los amplios horarios de trabajo de los progenitores.

Referente a esto, si atendemos a la posible implicación de la familia en los DE, se puede exponer que esta ayuda es la forma más común que tienen de involucrarse en la educación de sus descendientes (Regueiro et al., 2015; Valle et al., 2016). Existen diversos estudios que analizan la relación entre la implicación familiar en los DE y el rendimiento académico (Castro et al, 2015; Patall, Cooper y Civay, 2008; Regueiro et al., 2015; Suárez et al., 2014; Suárez, Regueiro, Tuero, Cerezo y Rodríguez, 2014; Valle et al., 2016), pero los resultados no llegan a ser claros, incluso encontrando discrepancias. Pero, ¿qué opina la comunidad educativa de Murcia sobre esta implicación?, ¿es beneficiosa?, ¿familias, docentes y alumnos tienen la misma percepción?

Dadas estas cuestiones, junto al conflicto que existe actualmente, se propone este estudio con el fin de indagar sobre la realidad existente en la Región de Murcia con respecto a este tópico.

\section{Conceptualización de los deberes escolares}

Antes de entrar en materia, resulta apropiado establecer una definición sobre qué son los DE, utilizando para este fin las definiciones de varios autores.

Tareas, deberes, etc., son muchos los términos que se han usado a lo largo de los años para hacer referencia a estas actividades. En cuanto a una posible definición, si se atiende a González, Lacasa y Albuquerque (1999, p. 22), son "un proceso de aprendizaje formal en un contexto no formal", es decir, actividades que buscan que el alumnado alcance el conocimiento formal; que es aquel que se desarrolla en los centros educativos, pero se pretende que sea adquirido en entornos diferentes a la escuela.

Asimismo, Cooper, citado por Amiama Ibarguren (2013, p. 5), establece que son aquellas "tareas asignadas por los profesores destinadas a llevarse a cabo durante las horas no escolares". Como se puede observar, el autor solo hace referencia a aquellas actividades que el docente prescribe para realizar fuera del horario escolar, por lo tanto, esta definición excluye todas aquellas que se realizan dentro del colegios.

Pero hay que matizar que este concepto ha ido volviéndose cada vez más complejo, implicando un gran abanico de variables como la dificultad, la adaptación a las características individuales o el tipo de actividades, y tal como establece Castro Valley Unified School District (2001, p. 1) "Los deberes pueden incluir una amplia diversidad de actividades de aprendizaje de los estudiantes relacionadas con el currículo y no deben ser exclusivamente actividades de papel y lápiz".

\section{La implicación de las familias en los deberes escolares}

Tras analizar el término de DE, es preciso tratar el otro eje en el que se centra esta investigación, y es la influencia que origina la participación de las familias en las tareas escolares. Esta variable es tan importante debido a que, en la actualidad, la implicación familiar en los centros y en el proceso de enseñanza-aprendizaje se considera como uno de los indicadores de la calidad de los sistemas educativos, porque son uno de los agentes clave para favorecer el éxito escolar (Vallespir, Rincón y Morey, 2016). 
Según investigaciones previas (Alonso, 2018; Baldivis Secondary College, 2014; Blasco, 2018; Castro Valley Unified School District, 2001) los progenitores tienen ciertas responsabilidades y obligaciones con respecto a los DE. Entre ellas se destaca ayudar a elaborar un horario de estudio adecuado que les permita tener tiempo tanto para las tareas académicas, como para el ocio. Asimismo, Alonso (2018) añade el proporcionar un espacio fijo, con luminosos, libre de ruidos y con el material necesario, donde se reduzcan las posibles distracciones para el escolar.

Patall, Cooper y Civay (2008) recogen que son muchos los estudios que abogan por que mostrar un interés activo por la educación y los DE supone grandes beneficios para estos. Por lo cual, ofrecer refuerzos positivos tras su realización permitirá aumentar la motivación hacia los estudios. En cambio, no es adecuado obligarlos a realizar la tarea, puesto que todo aspecto que sea impuesto suele hacer que el alumnado pierda el interés (Alonso, 2018). La mayor parte de los estudios se dirigen a conocer la visión solo de uno de los grupos implicados, dejando la familia al margen; por ello, con este estudio se pretende abordar todos los grupos de la comunidad educativa para saber qué papel consideran que tienen dentro de este ámbito de la educación.

A pesar de que estas responsabilidades se exponen con carácter general, hay que tener en cuenta que, en la actualidad, las familias son muy heterogéneas. Pudiendo encontrar con más asiduidad familias monoparentales, madres solteras o progenitores separados. Referente a esto, Hornby y Lafaele (2011) elaboraron un modelo que incluye los factores que suponen una barrera para las familias a la hora de participar en los centros, es decir, aquellos que crean una distancia considerable entre los aspectos positivos que se destacan en la teoría y la realidad en la práctica diaria. Dichos factores son:

- Factores relacionados con las familias: incluye las creencias sobre la implicación familiar, la etnia, el género, etc.

- Factores ligados a los escolares: como la edad o las necesidades educativas.

- Factores relacionados a la relación familia-docentes: diferencias entre lenguajes, metas o actitudes.

- Factores sociales: económicos, políticos o demográficos.

Como se observa, unas de las variables que influye es el nivel económico, así como los aspectos socio-educativos (Llevot y Bernad, 2015), observándose que los infantes que pertenecen a familias con un estatus económico superior reciben más deberes y tienen mayor facilidad de acceso a recursos educativos (Hernández Prados y Gil Noguera, 2018); igualmente, la participación de estas familias suele ser mayor, puesto que en muchas ocasiones los progenitores poseen estudios superiores y le dan mayor importancia al logro académico de sus hijos e hijas (Alonso y Román, 2014; Bailén y Polo, 2016).

Como se observa aspecto económico suele estar relacionado con el nivel educativo. Sobre ello, Fernández-Freire, Rodríguez-Ruiz y Martínez-González (2019) apuntan que los progenitores parecen implicarse más en los deberes cuando sus conocimientos son los adecuados para poder ayudarles. Aun así, lo importante no es que lo sepan explicar todo, sino como destaca Alonso (2018), que estos les ayuden y animen a resolver las tareas, intentado no intervenir si no es esencial. 
Relativo a lo anterior, uno de los fallos que comenten los familiares cuando ayudan es ofrecer la respuesta correcta o incluso realizar la actividad, lo cual pone trabas al aprendizaje de sus hijos e hijas (Suárez et al., 2012), ya que de acuerdo con Álvaro (2011) prestar una ayuda innecesaria puede derivar en escolares poco autónomos, inseguros e incapaces de alcanzar una madurez completa. Todo ello hace necesario que los progenitores actúen simplemente como guías, exponiendo su interés por las tareas, pero dejándoles el trabajo que les pertenece (Dirección Nacional de Currículo, 2018). Se subraya la importancia de mostrar interés en relación a los deberes, puesto que, según Valle et al., (2016) y Patall, Cooper y Civay (2008), las actitudes que presentan los alumnos hacia los DE están determinadas por la influencia de las familias; es decir, la importancia que estos les den a la tarea, repercutirá en la importancia otorgada por los escolares.

El tiempo libre que poseen los progenitores, es otro aspecto influyente, ya que según Fraguela-Vale, Lorenzo-Castiñeiras, Merelas-Iglesias y Varela-Garrote (2013) existen discrepancias en su distribución según las características socioculturales. La transformación de la organización familiar que se ha ido experimentando a lo largo de los últimos años, ha originado que se haya reestructurado el tiempo libre de los infantes, ya que los progenitores no pueden pasarlo con ellos (Fraguela-Vale, Lorenzo-Castiñeiras y Varela-Garrote, 2011). Esto implica que, los estudiantes de familias económicamente superiores salgan favorecidos pues, aunque sus familias trabajen, pueden permitirse academias especializadas.

Asimismo, es relevante atender a que si realmente esta ayuda parental beneficia al desarrollo escolar. Álvarez et al. (2015) afirma que los resultados de los estudios indican que la familia influye fundamentalmente sobre las condiciones personales de los estudiantes, variables que están directamente relacionadas con el rendimiento académico. Coincidiendo con lo expuesto por Lastre, López y Alcázar (2018) en que hay "correlación estadísticamente significativa entre el apoyo familiar y el rendimiento académico", afirmación que se puede apoyar en el estudio de Balli, Demo, y Wedman (1998), donde se establece que el 95\% de los alumnos rinden mejor cuando obtiene ayuda de sus progenitores.

Otro aspecto que se ve beneficiado, es la comunicación entre progenitores y sus hijos e hijas (Blazer, 2009). Al tener la oportunidad de pasar más tiempo juntos y tratar temas de interés mutuos hace que se cree un vínculo más fuerte entre ellos. Además, se expone que estas actividades permiten a las familias contribuir en el proceso educativo, dándoles la oportunidad de conocer qué es lo que están trabajando cada día en el colegio. Sin embargo, no todo son beneficios, sino que en muchas ocasiones las expectativas creadas por los progenitores o la competición entre el alumnado crea una ansiedad a los discentes que influye negativamente es su motivación y en su rendimiento (Rosário, Mourão, Núñez, González-Pienda y Solano, 2006).

\section{Método}

Este trabajo responde a un enfoque metodológico cuantitativo, tratándose de un estudio de encuesta, de carácter descriptivo y transversal (Navarro, Jiménez, Rappoport y Thoilliez, 2017). 


\section{Objetivos}

El objetivo principal de este trabajo es determinar las percepciones que tienen los alumnos del segundo tramo de Educación Primaria, sus familias y maestros, pertenecientes a centros de la Región de Murcia, sobre la influencia que tiene la implicación de las familias en la realización de los deberes. En particular se pretenden estos tres objetivos específicos:

1. Valorar la opinión de los estudiantes sobre la influencia de la implicación de sus progenitores en la realización de los deberes escolares.

2. Analizar el punto de vista de las familias sobre la influencia de la participación familiar en los deberes.

3. Determinar la percepción de los docentes respecto a cómo influye la implicación familiar en las tareas para casa.

\section{Participantes}

Los participantes de este trabajo están conformados por el alumnado de Educación Primaria de nueve centros educativos de la Región de Murcia, así como por sus familias y maestros. La muestra se eligió por un criterio de disponibilidad siendo un total de 503 participantes.

El procedimiento que se realizó para la selección de la muestra es no probabilístico e intencional. Esto es así puesto que no son escogidos al azar, sino que todos cumplen una serie de criterios, destacando entre ellos la accesibilidad de las autoras a los distintos centros, así como la voluntariedad de la comunidad educativa a cumplimentar los cuestionarios.

En referencia al colectivo del alumnado se ha conseguido una muestra de 259 participantes. Para tener un conocimiento más fiel de este grupo, se procede a describir las características de la muestra productora de los datos que han sido obtenidos mediante preguntas de tipo académico. Del alumnado que conforman esta muestra el 63.7\% ( $n$ $=165)$ está escolarizado en un centro privado/concertado, mientras que el $36.7 \%$ ( $n=$ 94) acude a un centro de titularidad pública, destacando que la proporción de colegios de la región es de $75 \%$ públicos y $25 \%$ privados/concertados; habiendo acudido a siete colegios públicos y a dos privados.

Asimismo, se destaca que este conjunto está bastante equilibrado en relación al sexo, puesto que el $50.19 \%(n=130)$ de ellos son chicas y el $49.81 \%(n=129)$ chicos. Igualmente, es importante matizar que todo el alumnado pertenece al segundo tramo de Educación Primaria, siendo casi el 41\% $(n=106)$ alumnado de sexto curso, los cuales están muy igualados con el alumnado de quinto curso que conforma casi un $38 \%$ ( $n$ $=98)$ de la población y, por último, el $21.24 \%(n=55)$ restante son alumnos de cuarto.

La muestra de familias está compuesta por 163 miembros. En un primer momento se invitó a participar a 259 familias (muestra invitada) que coincide con los alumnos del estudio. Por lo tanto, se ha producido una mortandad experimental del $37.07 \%$ ( $n=$ 96). Atendiendo a los datos recogidos mediante las preguntas de tipo sociodemográficos se determina que el $79.1 \%(n=129)$ de los cuestionarios los ha realizado la madre, el $19.6 \%(n=32)$ fue el padre y solo un poco más del $1 \%(n=2)$ fue completado por un miembro diferente. 
Los familiares tienen una edad media de 41.28 años $(S d=5.231)$, oscilando entre 18 y 58 . De igual manera, la media referente al número de hijos e hijas por familia es de $1.89(S d=0.661)$, cuya amplitud se encontraba entre 1 y 4 hijos e hijas Concluyendo que el $43.56 \%(n=71)$ tienen estudios superiores; y que el 22.08\% $(n=36)$ están en situación de paro laboral.

El último colectivo está compuesto por 81 docentes. Se conoce que el 51.9\% $(n=42)$ de ellos pertenecen a centros de titularidad pública, mientras que el resto trabaja en centros privados/concertados. Por otra parte, en referencia a la situación administrativa de los maestros de los centros públicos el $38.3 \%(n=31)$ son funcionarios, es decir, tienen plaza fija en la función docente, mientras que algo menos del 14\% $(n=11)$ son interinos. Asimismo, más de las tres cuartas partes de los maestros son mujeres, siendo solo el $21 \%(n=17)$ hombres. Por último, referente a la edad, se observa que la media se encuentra en 38.93 ( $S d=10.061)$, oscilando entre los 23 y los 64 años.

\section{Instrumentos}

Los instrumentos que se emplearon fueron tres cuestionarios estructurados dirigidos a estudiantes, familias y docentes. Destacar que se diseñaron ad hoc para una investigación más amplia. Los reactivos que nos aportaban la información necesaria para comprender la concepción de la comunidad educativa en materia de DE conformaron una escala tipo Likert con cinco valores, donde el uno significaba totalmente en desacuerdo y el cinco totalmente de acuerdo.

El diseño de estos cuestionarios entrañó una revisión bibliográfica exhaustiva en torno a esta temática que supuso el análisis teórico-conceptual de las fuentes y de otros instrumentos ya creados (Baldivis Secondary College, 2014; Blazer, 2009, González Felipe y Reparaz Abaitua, 2012; Valle et al., 2015; Alonso, 2018). Esto permitió la identificación de las variables latentes o constructos más relevantes de la dimensión implicación familiar y DE. Tras ello, se procedió a la elaboración de los cuestionarios atendiendo a las características generales de los encuestados.

La primera propuesta fue revisada por dos jueces expertos, que valoraron cualitativamente la adecuación, claridad, pertinencia y relevancia de los reactivos, proponiendo mejoras que fueron incorporadas a las versiones definitivas. Esto dio lugar al cuestionario de percepción de los alumnos sobre los deberes (CUDE-A), al cuestionario de percepción de las familias sobre los deberes (CUDE-F) y al cuestionario de percepción de los maestros sobre los deberes (CUDE-M), empleando para esta investigación solo la dimensión de los cuestionarios referida a la implicación familiar. Destacar que los ítems de los tres cuestionarios guardan cierta similitud, variando simplemente la formulación de la mayor parte de ellos, por ejemplo, uno de los ítems dirigidos a los escolares sería "que mi familia me ayuden con los deberes es bueno para mí"; a las familias "ayudar a su hijo/a en los deberes es beneficioso"; y a los docentes "la ayuda de las familias en los deberes beneficia a los alumnos"

Los análisis de consistencia interna de las escalas referidas a la implicación familiar, realizados mediante el modelo Alpha de Cronbach evidencian que se presenta pobre la versión destinada a los alumnos, cuestionable la referida a sus familias, y aceptable la de los maestros (George y Mallery, 2003) (véase Tabla 1). 
Tabla 1

Consistencia interna $\alpha$ de Cronbach

\begin{tabular}{cccc}
\hline Cuestionario & n. ${ }^{\mathbf{0}}$ de elementos & $\begin{array}{c}\text { alpha de } \\
\text { Cronbach }\end{array}$ & $\begin{array}{c}\text { n.o de componentes } \\
\text { principales }\end{array}$ \\
\hline CUDE-A & 10 & .55 & 5 \\
\hline CUDE-F & 9 & .62 & 6 \\
\hline CUDE-M & 11 & .72 & 6 \\
\hline
\end{tabular}

Por su parte, la validez del constructo se ha analizado mediante un Análisis Factorial Exploratorio (AFE) utilizando el criterio de Kaiser para la extracción la extracción de los componentes principales y el método varimax para la rotación de la matriz de componentes principales. Previamente, los coeficientes Kaiser-Meyer-Olkin y la prueba de esfericidad de Barlett han evidenciado la adecuación de los AFE y, por ende, de los modelos factoriales que se generan (KMO entre .50 y .75, $p<.05)$. La información contenida en a Tabla 2 indica que las escalas gozan de la validez de constructo exigida en estudios de esta naturaleza.

Tabla 2

Validez del constructo

\section{Cuestionario}

CUDE-A
$\%$ de varianza total explicada

$21.05 \%$ sobre un $64.04 \%$ de la varianza del instrumento total

$30.55 \%$ sobre un $70.32 \%$ de la varianza del instrumento total

CUDE-F

\section{Procedimiento de recogida y análisis de datos}

El primer paso del procedimiento de recogida de datos fue acudir presencialmente a los centros escolares para tener un primer contacto con los miembros del equipo directivo. Esta reunión inicial tenía como fin explicarles con rigurosidad el objetivo de la investigación, así como los instrumentos a utilizar y el tiempo que 
ello conllevaría al centro. Seguidamente, se le proponía su participación en el estudio, entregándoles los consentimientos informados para cada una de las partes y, en el caso de aceptar, se establecía el día en el que se aplicarían los cuestionarios. Destacando, además, que se cuenta con un informe favorable de la Comisión Ética de la Universidad de Murcia.

En el segundo contacto se realizaron tres acciones. En primer lugar, se entregaron los cuestionarios de los docentes a los miembros del equipo directivo, para que estos se los hicieran llegar. En segundo lugar, se procedió a la aplicación de los cuestionarios de los alumnos de las clases que se habían acordado previamente. Y, finalmente, se entregó al alumnado participante los cuestionarios dirigidos a sus familias para que estos fueran cumplimentados en casa. Igualmente, se acordó con la dirección una última fecha para la recogida de los cuestionarios de los docentes y de las familias.

Recogidos los resultados, se realizó la elaboración de tres matrices de datos mediante el paquete estadístico IBM SPSS para Windows versión 19 y se procedió a realizar un análisis exploratorio de los datos con el fin de detectar y solventar errores. Posteriormente, se realizaron los análisis descriptivos de consistencia interna y el análisis factorial exploratorio.

\section{Resultados}

\section{Objetivo I.Valorar la opinión de los estudiantes sobre la influencia de la implica- ción de sus progenitores en la realización de los deberes escolares.}

Referente a este primer objetivo el 15.1\% $(n=39)$ de los estudiantes dicen que están totalmente de acuerdo con que sus familias les ayudaban con las tareas escolares. Asimismo, el 27\% $(n=70)$ expone estar totalmente de acuerdo con que la ayuda de los progenitores es beneficioso para ellos. El 12.4\% $(n=32)$ de los escolares asegura que la comunicación con las familias se mejorar al realizar deberes juntos. Frente a esto, el 37.8\% ( $n=98)$ está totalmente de acuerdo en que hacer tareas escolares con las familias hace que estos conozcan más lo que sucede en el centro escolar. En cuanto a si las familias saben ayudarlos con las actividades siempre, el 32.4\% $(n=84)$ dice que estaba totalmente de acuerdo con la afirmación.

Por su lado, el $12 \%(n=31)$ afirma estar totalmente de acuerdo en que sus padres y madres no tienen tiempo de ayudarlos con los deberes. Casi seis de cada 10 alumnos dicen estar totalmente de acuerdo en que los familiares les resuelven las dudas que les surgen, pero no les ofrecen la solución. Asimismo, el 23.9\% ( $n=$ 62) de la muestra establece estar totalmente de acuerdo con que sus progenitores conocen siempre los deberes que tienen que hacer. Igualmente, casi cuatro de cada 10 estudiantes dicen estar totalmente de acuerdo con que, si los familiares les otorgan importancia a los deberes, ellos también se la dan; $\mathrm{y}$, por último, solo $3.1 \%$ ( $n$ $=8$ ) totalmente en de acuerdo con que siempre le pide ayuda a la familia para la realización de la tarea. La Tabla 3 plasma los resultados recogidos sobre los ítems valorados en este objetivo. 
Tabla 3

Objetivo 1

\begin{tabular}{cccccccc}
\hline Ítem & $\begin{array}{c}\mathbf{1} \text { (muy en } \\
\text { desacuerdo) }\end{array}$ & $\mathbf{2}$ & $\mathbf{3}$ & $\mathbf{4}$ & $\begin{array}{c}\mathbf{5} \text { (muy de } \\
\text { acuerdo) }\end{array}$ & Media & SD \\
\hline 1 & $21.2 \%$ & $22.8 \%$ & $26.3 \%$ & $14.3 \%$ & $15.1 \%$ & 2.99 & 3.51 \\
\hline 2 & $20.5 \%$ & $17.4 \%$ & $20.5 \%$ & $14.7 \%$ & $27 \%$ & 3.10 & 1.49 \\
\hline 3 & $42.5 \%$ & $13.9 \%$ & $20.1 \%$ & $11.2 \%$ & $12.4 \%$ & 2.37 & 1.43 \\
\hline 4 & $13.1 \%$ & $6.6 \%$ & $20.1 \%$ & $22.4 \%$ & $37.8 \%$ & 3.65 & 1.38 \\
\hline 5 & $13.5 \%$ & $11.2 \%$ & $23.2 \%$ & $19.7 \%$ & $32.4 \%$ & 3.46 & 1.39 \\
\hline 6 & $30.1 \%$ & $20.1 \%$ & $25.9 \%$ & $12 \%$ & $12 \%$ & 2.56 & 1.35 \\
\hline 7 & $16.6 \%$ & $5.4 \%$ & $10.4 \%$ & $10.4 \%$ & $57.1 \%$ & 3.86 & 1.54 \\
\hline 8 & $29.7 \%$ & $13.5 \%$ & $19.3 \%$ & $13.5 \%$ & $23.9 \%$ & 2.88 & 1.55 \\
\hline 9 & $15.1 \%$ & $10 \%$ & $15.8 \%$ & $19.3 \%$ & $39.8 \%$ & 3.59 & 1.47 \\
\hline 10 & $49 \%$ & $26.6 \%$ & $15.8 \%$ & $5.4 \%$ & $3.1 \%$ & 1.87 & 1.06 \\
\hline
\end{tabular}

Objetivo 2.Analizar la influencia de la participación familiar en las tareas escolares, desde el punto de vista de las familias.

Si se continua con las opiniones de las familias, el 23.3\% $(n=38)$ de estas exponen que están totalmente de acuerdo en que ayudar con los deberes es beneficioso. En cuanto a que la realización de estos permite aumentar la comunicación con sus hijos e hijas, el 23.9\% $(n=39)$ aseguró que estar totalmente de acuerdo. Asimismo, el 21.5\% $(n=35)$ de la muestra dice estar totalmente de acuerdo con que los deberes favorecen la participación de las familias en los aspectos escolares. El 31.3\% $(n=51)$ dice que está totalmente de acuerdo con que tiene la preparación adecuada para ayudar con las tareas escolares.

Referente al tiempo disponible, se observa que algo más de 27 familiares están totalmente de acuerdo en que tienen tiempo suficiente para ayudar a sus hijos e hijas. El $33.1 \%(n=54)$ de la muestra está totalmente de acuerdo en que cuando les piden ayuda solo actúan como guía. De igual manera, algo más de cuatro de cada 10 familias dice que está totalmente de acuerdo en que estas tareas les permiten conocer lo que hace su hijo o hija en clase. Un poco más de la mitad de las familias encuestadas están totalmente de acuerdo, o bastante de acuerdo en que el grado de importancia que ellos le dan a los deberes repercute en el que le den sus hijos e hijas. Finalmente, el $9.8 \%$ ( $n=$ 16) de estos expone estar totalmente de acuerdo con que en ocasiones sus descendientes piden ayuda para realizar las actividades solo porque no tienen ganas de hacerlos. En la Tabla 4 se recogen los resultados referidos a las opiniones de las familias. 
Tabla 4

Objetivo 2

\begin{tabular}{cccccccc}
\hline Ítem & $\begin{array}{c}\mathbf{1} \text { (muy en } \\
\text { desacuerdo) }\end{array}$ & $\mathbf{2}$ & $\mathbf{3}$ & $\mathbf{4}$ & $\begin{array}{c}\mathbf{5} \text { (muy de } \\
\text { acuerdo) }\end{array}$ & Media & SD \\
\hline 1 & $9.2 \%$ & $6.7 \%$ & $35 \%$ & $25.8 \%$ & $23.3 \%$ & 3.47 & 1.19 \\
\hline 2 & $15.3 \%$ & $5.5 \%$ & $20.9 \%$ & $34.4 \%$ & $23.9 \%$ & 3.46 & 1.33 \\
\hline 3 & $8.6 \%$ & $6.1 \%$ & $31.3 \%$ & $32.5 \%$ & $21.5 \%$ & 3.52 & 1.51 \\
\hline 4 & $6.1 \%$ & $5.5 \%$ & $20.9 \%$ & $36.2 \%$ & $31.3 \%$ & 3.81 & 1.13 \\
\hline 5 & $27 \%$ & $14.1 \%$ & $27 \%$ & $15.3 \%$ & $16.6 \%$ & 2.80 & 1.42 \\
\hline 6 & $6.7 \%$ & $6.1 \%$ & $23.3 \%$ & $30.7 \%$ & $33.1 \%$ & 3.77 & 1.17 \\
\hline 7 & $4.3 \%$ & $4.3 \%$ & $12.9 \%$ & $35 \%$ & $43.6 \%$ & 4.09 & 1.06 \\
\hline 8 & $8 \%$ & $7.4 \%$ & $23.3 \%$ & $30.7 \%$ & $30.7 \%$ & 3.69 & 1.21 \\
\hline 9 & $41.1 \%$ & $16 \%$ & $15.3 \%$ & $17.8 \%$ & $9.8 \%$ & 2.39 & 1.42 \\
\hline
\end{tabular}

Objetivo 3. Determinar la percepción de los docentes respecto a cómo influye la implicación familiar en las tareas para casa.

Finalmente, atendiendo a los docentes, se observa que el 14.8\% $(n=12)$ de ellos está totalmente de acuerdo en que los familiares deben obligar a sus hijos e hijas a que hagan los deberes. En cuanto al nivel socioeconómico, algo más de cuatro de cada 10 docentes aseguran estar totalmente de acuerdo en que este influye en el grado de implicación de las familias. Igualmente, el $18.5 \%(n=15)$ de la muestra afirma estar totalmente de acuerdo con que la ayuda de los progenitores en las tareas es beneficiosa. De igual manera, algo más de 1 de cada diez docentes dice estar totalmente de acuerdo con que la realización de los deberes permite aumentar la comunicación entre familias e hijos e hijas.

Por otra parte, el $16 \%(n=13)$ de los docentes dicen estar totalmente de acuerdo en que las tareas favorecen la participación de las familias en los aspectos escolares. En lo concerniente a si los padres y madres tienen una preparación adecuada para ayudar a sus hijos, solo siete miembros del profesorado dicen estar totalmente de acuerdo. Igualmente, el $17.3 \%(n=14)$ asegura estar totalmente de acuerdo con que los progenitores tienen el tiempo suficiente para atender a los hijos e hijas con las tareas escolares. Asimismo, casi siete de cada 10 docentes exponen estar totalmente de acuerdo en que los familiares deben actuar como solo como un apoyo.

Casi tres cuartos de la muestra dicen estar totalmente de acuerdo, o bastante de acuerdo en que la realización de los deberes permite que los progenitores conozcan lo que se está dando en clase. De igual forma, el $42 \%(n=34)$ está totalmente de acuerdo con que el grado de importancia que le dan los progenitores a los deberes influye en 
el grado de importancia que le dan los hijos. Finalmente, el 23.5\% $(n=19)$ afirma estar totalmente de acuerdo con que, en ocasiones, los escolares piden ayuda a los familiares porque no tienen ganas de hacer las tareas. La Tabla 5 recoge los resultados de las percepciones de los docentes.

Tabla 5

\section{Objetivo 3}

\begin{tabular}{cccccccc}
\hline Ítem & $\begin{array}{c}\mathbf{1} \text { (muy en } \\
\text { desacuerdo) }\end{array}$ & $\mathbf{2}$ & $\mathbf{3}$ & $\mathbf{4}$ & $\begin{array}{c}\mathbf{5} \text { (muy de } \\
\text { acuerdo) }\end{array}$ & Media & SD \\
\hline 1 & $3.7 \%$ & $17.3 \%$ & $30.9 \%$ & $33.3 \%$ & $14.8 \%$ & 3.38 & 1.06 \\
\hline 2 & $3.7 \%$ & $11.1 \%$ & $11.1 \%$ & $32.1 \%$ & $42 \%$ & 3.98 & 1.15 \\
\hline 3 & $4.9 \%$ & $12.3 \%$ & $35.8 \%$ & $28.4 \%$ & $18.5 \%$ & 3.43 & 1.08 \\
\hline 4 & $9.9 \%$ & $13.6 \%$ & $35.8 \%$ & $25.9 \%$ & $14.8 \%$ & 3.22 & 1.16 \\
\hline 5 & $7.4 \%$ & $7.4 \%$ & $29.6 \%$ & $39.5 \%$ & $16 \%$ & 3.49 & 1.09 \\
\hline 6 & $8.6 \%$ & $35.8 \%$ & $46.9 \%$ & $8.6 \%$ & $0 \%$ & 2.56 & .76 \\
\hline 7 & $6.2 \%$ & $11.1 \%$ & $37 \%$ & $28.4 \%$ & $17.3 \%$ & 3.40 & 1.09 \\
\hline 8 & $0 \%$ & $2.5 \%$ & $4.9 \%$ & $24.7 \%$ & $67.9 \%$ & 4.58 & .71 \\
\hline 9 & $4.9 \%$ & $3.7 \%$ & $17.3 \%$ & $43.2 \%$ & $\%$ & 3.91 & 1.04 \\
\hline 10 & $2.5 \%$ & $1.2 \%$ & $18.5 \%$ & $35.8 \%$ & $42 \%$ & 4.14 & .93 \\
\hline 11 & $1.2 \%$ & $2.5 \%$ & $25.9 \%$ & $46.9 \%$ & $23.5 \%$ & 3.89 & .84 \\
\hline
\end{tabular}

\section{Discusión y conclusiones}

Finalizado el análisis de datos, se procede a destacar las conclusiones más relevantes obtenidas a través de este estudio:

- La mayor parte del alumnado expone que no recibe ayuda de sus progenitores, sin embargo, cree que recibir esa ayuda es beneficiosa para su formación.

- Los estudiantes afirman que sus familiares sí tienen tiempo de atenderlos con las tareas escolares y que siempre saben ayudarlos con los contenidos.

- Los familiares creen que los deberes mejoran la comunicación con sus hijos y que, además, les permiten saber lo que este está trabajando en la escuela cada día.

- Otro aspecto que remarcan los progenitores es que no disponen del tiempo suficiente para ayudar a sus hijos en estos aspectos académicos, lo cual entra en debate con lo que piensan los escolares. 
- Por su lado, los docentes creen que los familiares deben obligar a sus hijos e hijas a hacer las tareas y creen firmemente que el nivel socioeconómico de la familia influye en la implicación de estos en el ámbito escolar.

- Sin embargo, los mismos docentes no tienen una percepción clara sobre si los familiares tienen los conocimientos y el tiempo adecuado para dedicarles a sus hijos e hijas.

- Señalando finalmente que los docentes son los únicos que piensan que el alumnado pide ayuda innecesaria a sus progenitores cuando no tienen ganas de hacer las tareas.

Si prestamos atención a las conclusiones obtenidas en este estudio, se puede observar que, a pesar del conflicto que existe sobre los deberes tanto a nivel mundial, como nacional, en la muestra analizada en la Región de Murcia tiene percepciones bastantes positivas hacia los DE y hacia la implicación de las familias en esta materia.

Es cierto que en los resultados se encuentran discrepancias entre los distintos colectivos encuestados puesto que, aunque los alumnos piensan que sus progenitores tienen suficiente tiempo para dedicarles, los otros dos colectivos coinciden con Fraguela-Vale et al., (2011) cuando afirman que la organización del tiempo se ha transformado en uno de los principales problemas familiares, sobre todo para aquellas que cuentan con hijos e hijas, puesto que, como exponen Fraguela-Vale, Lorenzo-Castiñeiras, Merelas-Iglesias y Varela-Garrote (2013), la educación y escolarización de los hijos supone incluir en el día a día gran cantidad de tareas, como la ayuda en los deberes o distintas reuniones; aspecto que no siempre resulta sencillo.

De la misma manera, también se observan opiniones divergentes respecto a los conocimientos de los familiares, siendo los docentes los únicos que no tienen muy claro que los progenitores tengan el suficiente conocimiento y formación para ayudar a sus hijos. Por ello, se coincide con la Dirección Nacional de Currículo (2018) al establecer la necesidad de que la familia se comporte como apoyos y guías, dejando la formación a los docentes.

Sin embargo, Suarez et al. (2012) expone que la mayor parte de las familias piensan que su ayuda con los deberes es eficaz; a lo cual se añade que los escolares sienten que su rendimiento académico es superior cuando los progenitores le prestan su ayuda, lo cual concuerda totalmente con los resultados recogidos en esta muestra. Por lo cual, siguiendo a estos autores sería interesante, por un lado, llevar a cabo la formación desde las universidades en materia de colaboración familia-escuela, con el fin de que los docentes sean capaces de orientar a los familiares en el proceso de enseñanza de sus descendientes; y, por otro lado, la creación de escuelas de familias para potenciar esa colaboración desde la perspectiva familiar.

A pesar de estas discrepancias, la mayor parte de la muestra están de acuerdo en que la implicación familiar incide de forma positiva en la realización de tareas escolares, ya que como exponen Suárez et al. (2012) el rendimiento de los escolares se ve gratamente favorecido cuando reciben la ayuda de sus progenitores.

Exponer también que las conclusiones de este trabajo no solo son especialmente relevantes para que nuestros órganos de gobierno, los centros y la comunidad educativa cuente con información contrastada para regular los DE, sino también para instar 
a las universidades a que incluyan en los planes de estudios de los grados y másteres en educación formación específica sobre los deberes. Además, se trata de una investigación que ha generado información de los tres agentes educativos más relevantes (alumnado, familias y docentes) mediante tres cuestionarios paralelos diseñados ad hoc que han sido sometidos a un primer proceso de validación y que podrán ser utilizados y mejorados en futuras investigaciones.

Finalmente, es preciso señalar que, entre las limitaciones de este trabajo, se encuentran la gran mortandad experimental de los familiares o que la muestra no llega a ser significativa para la región. Asimismo, destacar que con este estudio se pretende abrir nuevas líneas de investigación relacionadas con el tema de los DE como: elaboración de guías para familias y docentes sobre cómo mejorar la colaboración familia-escuela; estilos de aprendizaje y su relación con los DE; estilos docentes y su relación con los DE.

\section{Referencias}

Alonso, J., \& Román, J. M. (2014). Nivel sociocultural, prácticas educativas familiares y autoestima de los hijos en edades tempranas. Revista de Investigación Educativa, 32(1), 187-202. doi: http://dx.doi.org/10.6018/rie.32.1.173421

Alonso, M. B. (2018). El puzzle de los deberes. Recuperado de https://www.comunidad. madrid/file/126823/download?token=wjuQlxyH

Álvarez, A., Suárez, N., Tuero, E., Ñúñez, J. C., Valle, A., \& Regueiro, B. (2015). Implicación familiar, autoconcepto del adolescente y rendimiento académico. European Journal of Investigation in Health, Psychology and Education, 5(3), 293-311.

Álvaro, P. (2011). La sobreprotección infantil (Tesis doctoral, Universidad Pedagógica Nacional). Recuperada de http://200.23.113.51/pdf/28727.pdf

Amiama Ibarguren, J. F. (2013). Análisis de los deberes escolares en la E.S.O. y exploración de un espacio colaborativo entre profesorado y familias en la Comunidad Autónoma Vasca. (Tesis doctoral, Universidad del País Vasco). Recuperado de https://addi.ehu.es/bitstream/10810/12178/1/amiama\%20ibarguren.pdf

Asamblea Regional de Murcia (2016). Moción sobre regulación y racionalización de los deberes escolares en horario extraescolar. Murcia, España: BOAR. Recuperado de: http://hermes. asambleamurcia.es/documentos/pdfs/boar/Boar.09/160419.043.pdf

Bailén, E., \& Polo, I. (2016). Deberes escolares: el reflejo de un sistema educativo. Revista Avances en supervisión educativa, 25, 1-36. doi: https://doi.org/10.23824/ase.v0i25.543

Baldivis Secondary College (2014). Homework and Study Policy. Recuperado de http:// baldivissc.wa.edu.au/wp-content/uploads/2014/01/Homework-and-Study-Policy.pdf

Balli, S. J., Demo, D. H., \& Wedman, J. F. (1998). Family involvement with children's homework: An intervention in the middle grades. Family Relations, 47, 149-157.

Blasco, J. (2018). ¿Los programas para fomentar la implicación parental en la educación sirven para mejorar el rendimiento escolar? Fundación Jaume Bofill e Institut Catalá d'Avaluació de Polítiques Públiques. Recuperado de http://www.ivalua.cat/documents/1/10_10_2018_13_51_55_Que_funciona_11_castella.pdf

Blazer, C. (2009). Literature Review: Homework. Miami: Miami Dade County Public Schools. Recuperado de http://drs.dadeschools.net/LiteratureReviews/Homework.pdf 
Castro Valley Unified School District (2001). Homework. Recuperado de http://stanton. cv.k12.ca.us/AR\%206154\%20\%20Homework.pdf

Castro, M., Expósito-Casas, E., López-Martín, E., Lizasoain, L., Navarro-Asencio, E., \& Gaviria, J. L. (2015). Parental involvement on student academic achievement: A metaanalysis. Educational Research Review, 14, 33-46.

Dirección Nacional de Currículo (2018). Guía de tareas escolares. Quito, Educador: Ministerio de Educación del Educador.

Fernández-Freire, L., Rodríguez-Ruiz, B., \& Martínez-González, R. (2019). Padres y madres ante las tareas escolares: La visión del profesorado. Aula abierta, 48(1), 77-84.

Fraguela-Vale, R., Lorenzo-Castiñeiras, J. J., \& Varela-Garrote, L. (2011). Escuela, familias y ocio en la conciliación de los tiempos cotidianos de la infancia. Revista de Investigación Educativa, 29 (2), 429-446.

Fraguela-Vale, R., Lorenzo-Castiñeiras, J. J., Merelas-Iglesias, T., \& Varela-Garrote, L. (2013). Tiempos escolares y conciliación: análisis de familias con hijos en educación secundaria obligatoria (12-16 años). Revista de Investigación Educativa, 31(2), 431-446. doi: 10.6018/rie.31.2.167001

Generalitat Valenciana (2018). Ley 26/2018, de 21 de diciembre, de derechos y garantías infancia y la adolescencia. Valencia, España: DOGV. Recuperado de: https://www. dogv.gva.es/portal/ficha_disposicion_pc.jsp?sig=011597/2018\&L=1

George, D., \& Mallery, P. (2003). SPSS for Windows step by step: A simple guide and reference. 11.0 update (4thed.). Boston: Allyn \& Bacon.

Gobierno de Aragón (2018). Orden ECD/2146/2018, de 28 de diciembre, por la que se convoca a los centros docentes sostenidos con fondos públicos de Educación Infantil, Primaria y Secundaria y en centros de Educación Especial de la Comunidad Autónoma de Aragón para el desarrollo del proceso de reflexión en torno a las tareas escolares en el proceso de aprendizaje. Aragón, España: Boletín Oficial de Aragón. Recuperado de http://www. educaragon.org/noticias/noticias.asp?idNoticia $=14195$

González Felipe, P., \& Reparaz Abaitua, C. (2012). La opinión de los estudiantes sobre las tareas escolares. Navarra: Consejo Escolar de Navarra. Recuperado de https://www. navarra.es/NR/rdonlyres/D096F1EB-B08C-4554-82D5-C402A27850AF/221905/2305 12ed70estudio.pdf

González, P., Lacasa, P., \& Albuquerque, M. (1999). Los deberes escolares: ¿qué hay y qué buscamos? Cultura y Educación, 11(1), 21-36. doi: 10.1174/113564002320584817

Hernández Prados, M. A., \& Gil Noguera, J. A. (2018). Los deberes a examen. Riesgos y oportunidades. En J. C. Martínez Coll (Coord.), III Congreso online internacional sobre la educación del siglo XXI (pp. 116-129). Recuperado de https://www.eumed. net/actas/18/educacion/9-los-deberes-a-examen-riesgos.pdf

Hornby, G., \& Lafaele, R. (2011). Barriers to parental involvement in education: an explanatory model. Educational Review, 63, 37-52. doi: https://doi.org/10.1080/0013 1911.2010.488049

Jiménez Becerra, A. (2010). El devenir de las tareas escolares y la emergencia de la infancia contemporánea: Una arqueología histórica en Colombia entre 1968 y 2006. Pedagogía y Saberes, 33, 51-61. Recuperado de http://revistas.pedagogica.edu.co/index. php/PYS/article/view/1247/1237 
Lastre, K., López, L., \& Alcázar, C. (2018). Relación entre apoyo familiar y el rendimiento académico en estudiantes colombianos de educación primaria. Psicogente, 21(39), 102-115. http://doi.org/10.17081/psico.21.39.2825

Llevot, N., \& Bernad, O. (2015). La participación de las familias en la escuela: factores clave. Revista de la Asociación de Sociología de la Educación, 8(1), 57-70.

Navarro, N., Jiménez, E., Rappoport, S., \& Thoeilliez, B. (2017). Fundamentos de la investigación y la innovación educativa. La Rioja: Universidad Internacional de La Rioja S.A.

Patall, E. A., Cooper, H., \& Robinson, J. C. (2008). Parent involvement in homework: A research synthesis. Review of Educational Research, 78(4), 1039-1101.

Regueiro, B., Rodriguez, S., Piñeiro, I., Estévez, I., Ferradás, M., \& Suárez, N. (2015). Diferencias en la percepción de la implicación parental en los deberes escolares en función del nivel de motivación de los estudiantes. European Journal of Investigation in Health, Psychology and Education, 5(3), 313-323.

Rosário, P., Mourão, R., Núñez, J. C., González-Pienda, J. A., \& Solano, P. (2006). Escuela-familia: ¿es posible una relación recíproca y positiva? Papeles del Psicólogo, 27(3), 171-179.

Suárez, N., Fernández, E., Cerezo, R., Rodríguez, C., Rosári, P., \& Núñez, J. C. (2012). Tareas para casa, implicación familiar y rendimiento académico. Aula Abierta, 40(1), 73-84. Recuperado de http://dspace.sheol.uniovi.es/dspace/bitstream/10651/17589/1/ AulaAbierta.2012.40.1.73-84.pdf

Suárez, N., Núñez, J. C., Vallejo, G., Cerezo, R., Regueiro, B., \& Rosário, P. (2014). Tareas para casa, rendimiento académico e implicación de padres y profesores. International Journal of Developmental and Educational Psychology, 7(1), 417-423.

Suárez, N., Regueiro, B., Tuero, E., Cerezo, R., \& Rodríguez, C. (2014). La implicación familiar en el ámbito educativo como herramienta para trabajar el éxito académico. Revista de Psicología y Educación, 9(2), 83-93.

Valle, A., Pan, I., Nuuñez, J.C., Rosário, P., Rodríguez, S., \& Regueiro, B. (2015). Deberes escolares y rendimiento académico en Educación Primaria. Anales de Psicología, 31(2), 562-569. doi:10.6018/analesps.31.2.171131

Valle, A., Regueiro, B., Ñuñez, J.C., Suárez, N., Freire, C., \& Ferradás, M. (2016). Percepción de la implicación parental en los deberes escolares y rendimiento académico en estudiantes de Secundaria. Revista española de pedagogía, 265, 481-498.

Vallespir, J., Rincón, J. C., \& Morey, M. (2016). La participación de las familias en el Consejo Escolar y la formación del profesorado. Revista Electrónica Interuniversitaria de Formación del Profesorado, 19(1), 31-45.

Fecha de recepción: 22 de febrero de 2019.

Fecha de revisión: 7 de marzo de 2019.

Fecha de aceptación: 17 de diciembre de 2019. 